\title{
Unveiling the real dynamics of transmission of COVID-19 in Malaysia without incarcerated clusters: a modelling study
}

Kian Boon Law ( $\sim$ kblaw@crc.gov.my )

Institute for Clinical Research, Malaysia https://orcid.org/0000-0002-1175-1307

Kalaiarasu M Peariasamy

Institute for Clinical Research, Malaysia

\section{Research Article}

Keywords: COVID-19, SIR model, incarcerated population, dynamics of transmission, prison

Posted Date: October 23rd, 2020

DOI: https://doi.org/10.21203/rs.3.rs-97023/v1

License: (c) (i) This work is licensed under a Creative Commons Attribution 4.0 International License.

Read Full License 


\title{
Unveiling the real dynamics of transmission of COVID-19 in Malaysia without incarcerated clusters: a modelling study
}

\section{Authors:}

Law Kian Boon, Kalaiarasu M. Peariasamy

\begin{abstract}
Affiliations
Institute for Clinical Research, National Institutes of Health, Ministry of Health Malaysia

Corresponding author:
\end{abstract}

Law Kian Boon,

\begin{abstract}
Introduction: The latest outbreak of COVID-19 in Malaysia emerged in early September and had two determinants. First, it involved incarcerated populations from four prisons located in Sabah, Kedah and Penang states. Second, the Sabah state by-election campaigns accelerated the spread of COVID-19 in the state and across the South China Sea into the west Malaysia. The emergence of multiple incarcerated clusters at different time points may shadow the real dynamics of transmission of COVID-19 in the community and lead to inaccurate interpretation and conclusion. The study aimed to reveal the real spreading pattern of COVID-19 by excluding incarcerated clusters in the modelling.

Methodology: We extended the susceptible-infectious-removed (SIR) model to include an additional class for non-isolated active cases, which was assumed to impel the transmission of COVID-19 in the community. The model was fitted to actual total and removed cases for estimation of duration of transmission and hospitalization. The parameters were then applied to model the transmission for COVID-19 in the community.

Results: The presence of incarcerated clusters shadowed the dynamics of transmission of COVID19 with a lower reproduction number $(\widehat{\mathrm{R}})$ of 2.0. The proportion of non-isolated active cases increased slowly from $49.4 \%$ on 1 September 2020 to $60.3 \%$ on 8 October 2020. In the absence of incarcerated clusters, the dynamics of transmission of COVID-19 appeared differently with a higher $\widehat{\mathrm{R}}$ of 2.3 . The proportion of non-isolated active cases increased tremendously from $22.1 \%$ on 1 September 2020 to $63.7 \%$ on 8 October 2020. The tremendous increase of non-isolated active cases impelled the dynamics of transmission of COVID-19 in the community following the Sabah state byelection campaigns and more inter-state travels.

Conclusion: The inclusion of incarcerated clusters shadowed the dynamics of transmission of COVID19 in the community with a lower transmission rate, which might lead to wrong interpretation of the dynamics of transmission in the community.
\end{abstract}

Keywords: COVID-19, SIR model, incarcerated population, dynamics of transmission, prison 


\section{Introduction}

The latest outbreak of coronavirus disease (COVID-19) in Malaysia emerged in early September 2020 and involved mostly prisoners from two prisons located in Lahad Datu and Tawau, Sabah (collectively referred as the Benteng LD cluster). Later, the outbreak accelerated in the state through series of by-election campaigns, public rallies and frequent inter-strict travels following the dissolve of the Sabah State Legislative Assembly on 30 July 2020. ${ }^{1}$ Not only that, the outbreak of COVID-19 in Sabah had crossed the South China Sea and entered into the west Malaysia, evidenced by over 350 cases of COVID-19 across different states found to have a history of travelling to Sabah since 20 September $2020 .^{2}$

As Malaysia was still under the Movement Control Order (MCO), by-election campaigns, public rallies and home visits were supposed to carry on under a specific standard operating procedure (SOP) imposed by the Electoral Commission (SPR). Unfortunately, many campaigners and attendees were found not complying with the SOP, therefore impelled the widespread of COVID19. ${ }^{1}$ On 6 October 2020, the Prime Minister announced targeted enhanced MCO (TEMCO) to be imposed on several localities to contain the spread of COVID-19, especially in Sabah. ${ }^{3}$ Toward the end of Benteng LD cluster, another major outbreak of COVID-19 occurred in Alor Setar prison following the death of a 46-year-old man who had worked at the prison (referred as the Tembok cluster). As of 8 October 2020, the Tembok cluster has recorded at least 1200 cases of COVID-19 out of 5149 individuals screened (23\%), with 4 cases admitted to ICU and one death. ${ }^{2}$

Prisoners and detainees were marginalized population and vulnerable to COVID-19., ${ }^{4,5}$ They faced an exceptionally high risk of infection due to an overcrowding and close confinement, insufficient sanitation and ventilation, and higher mortality due to higher rate of communicable illnesses. ${ }^{6,7}$ However, those clusters posted a minimal threat to the transmission in the community, as compared with massive gathering events such as by-election campaigns.

The emergence of multiple incarcerated clusters at different time points may introduce variation into the epidemic curve of COVID-19. The real dynamics of transmission of COVID-19 in the community could be shadowed with misleading transmissibility, and lead to inaccurate interpretation and conclusion. Therefore, it was crucial to examine the shadowing effect of incarcerated clusters to the dynamics of transmission of COVID-19 in the community.

The study aimed to reveal the real spreading pattern of COVID-19 by excluding incarcerated clusters in the modelling. We would examine the confounding effect of multiple incarcerated clusters to the transmission of COVID-19 in the community. We extended the model to include an additional class for non-isolated active cases which was assumed to be the determinant for transmission of COVID-19 in the community.

\section{Methodology}

\section{Data source}

The Ministry of Health (MOH) Malaysia updates data on COVID-19 daily via a centralized website at https://kpkesihatan.com/. ${ }^{2}$ Those data include total cases, new cases, active cases,

recovered case and death cases for COVID-19. Break-ups of cases according to different states and clusters are documented separately and only for daily cases. Clusters involving mostly prisoners or 
detainees were Benteng LD cluster in Sabah, Tembok cluster in Kedah and Reman cluster in Pulau Pinang. The affected population was estimated to be 3000 for the Benteng LD cluster and 1800 for the Tembok cluster. ${ }^{2}$

A case was confirmed when being tested positive for COVID-19 with a real-time reverse transcriptase-polymerase chain reaction (rRT-PCR) test. In Malaysia, positive cases were all admitted to designated hospitals immediately for isolation and monitored for potential signs of further deterioration. Recovered cases were those discharged and tested negative for COVID-19. Active cases were those remained infectious for COVID-19, hospitalized and isolated. Removed cases consisted of recovered cases and death cases. To date, isolation of active cases remains an effective measure to prevent the spread of coronavirus in a community in the absence of effective antiviral therapies.

\section{Model structure}

A susceptible-infectious-removed (SIR) compartmental model was extended to include an additional class for non-isolated infectious cases. The non-isolated infectious class consisted of active cases or individuals of COVID-19 that were yet to be confirmed and isolated. These individuals were assumed to be the determinant for the transmission of COVID-19 in the community in the latest outbreak.

Three factors were considered for modelling. First, the reported trendline of COVID-19 was shadowed and confounded with the inclusion of incarcerated clusters at different time points, therefore might not accurately reflect the real dynamics of transmission of COVID-19 in the community. Second, active cases were individuals hospitalized for isolation and monitored in a hospital environment. Those individuals would not cause transmission during their isolation period and did not contribute to the spread of COVID-19 in the community. A class had to be created to account for the transmission of COVID-19 in the community by non-isolated active cases. At this stage, the impact of imported cases was insignificant due to a relatively small number of cases as compared to the total confirmed cases locally.

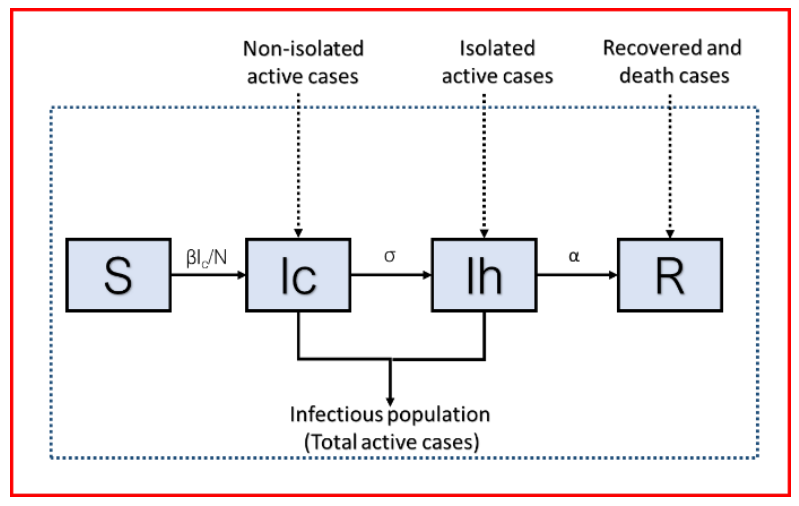

\section{Figure 1: Model structure}

The infectious class was divided into non-isolated infectious and isolated infectious. Non-isolated infectious was assumed to be the determinant for the spread of coronavirus in the community.

The extended SIR model was illustrated in Figure 1. The population, $N$ was divided into four classes, namely susceptible (S), non-isolated infectious (IC), isolated infectious (Ih), and removed (R). The infectious (I) compartment was extended to include individuals who were infectious but yet to be 
isolated (IC), and individuals who were tested positive for COVID-19 and hospitalized for isolation (Ih). We assumed that the proportion of non-isolated infection cases, given by Ic/N, was the only source of transmission for COVID-19 in the community.

The rate of transmission was given by $\beta \mathrm{lc} / \mathrm{N}$, with $\beta$ denotes the coefficient of transmission. The transition of individuals from Ic to Ih was controlled by a transition coefficient, $\sigma$, which was defined as one over the duration of transmission before being isolated. The transition of individuals from Ih to R was controlled by another transition coefficient, $\alpha$, which was defined as one over the duration of isolation or hospitalization. Both durations could be estimated by fitting the $\mathrm{lh}$ and $\mathrm{R}$ classes to the actual active or total, and removed cases over time.

Ordinary differential equations for the model were established as below:

$$
\begin{aligned}
& \frac{\mathrm{d} S}{\mathrm{dt}}=-\frac{\beta I c S}{\mathrm{~N}} \\
& \frac{\mathrm{dIc}}{\mathrm{dt}}=\frac{\beta I c S}{\mathrm{~N}}-\sigma \mathrm{Ic} \\
& \frac{\mathrm{dIh}}{\mathrm{dt}}=\sigma \mathrm{Ic}-\alpha \mathrm{Ih} \\
& \frac{\mathrm{dR}}{\mathrm{dt}}=\alpha \mathrm{Ih}
\end{aligned}
$$

Cumulative confirmed cases that had been hospitalized for isolation was,

$$
\frac{\mathrm{d} \hat{\mathrm{Ih}}}{\mathrm{dt}}=\sigma \mathrm{Ic}
$$

Assuming the source of transmission was non-isolated active cases of COVID-19 in the community, the reproduction number $(\mathrm{Rp})$ of the transmission based on the model was

$$
\widehat{\mathrm{R}}=\frac{\beta}{\sigma} \quad \text { or } \beta \times \text { duration of transmission }
$$

The initial phase of an infectious disease outbreak can be characterized by a basic reproduction number $\left(\widehat{R}_{0}\right)$, until the introduction of control measures that significantly change population interaction and contact rate.

\section{Model fitting strategy}

The model was first fitted to total confirmed and removed cases of COVID-19 with inclusion incarcerated clusters for the estimation of duration of transmission (before hospitalization) and isolation (after hospitalization). Assuming the duration of transmission and hospitalization remained, those parameters were then applied for the projection of active and removed cases in the absence of incarcerated clusters.

\section{Model assessment and validation}

Projections of the model were made based on actual cases from 1 September 2020 to 8 October 2020. Model fittings were assessed using mean absolute percent error (MAPE), calculated as the difference between projection and actual cases. Projections were validated using actual cases reported from 9 October to 15 October 2020. 


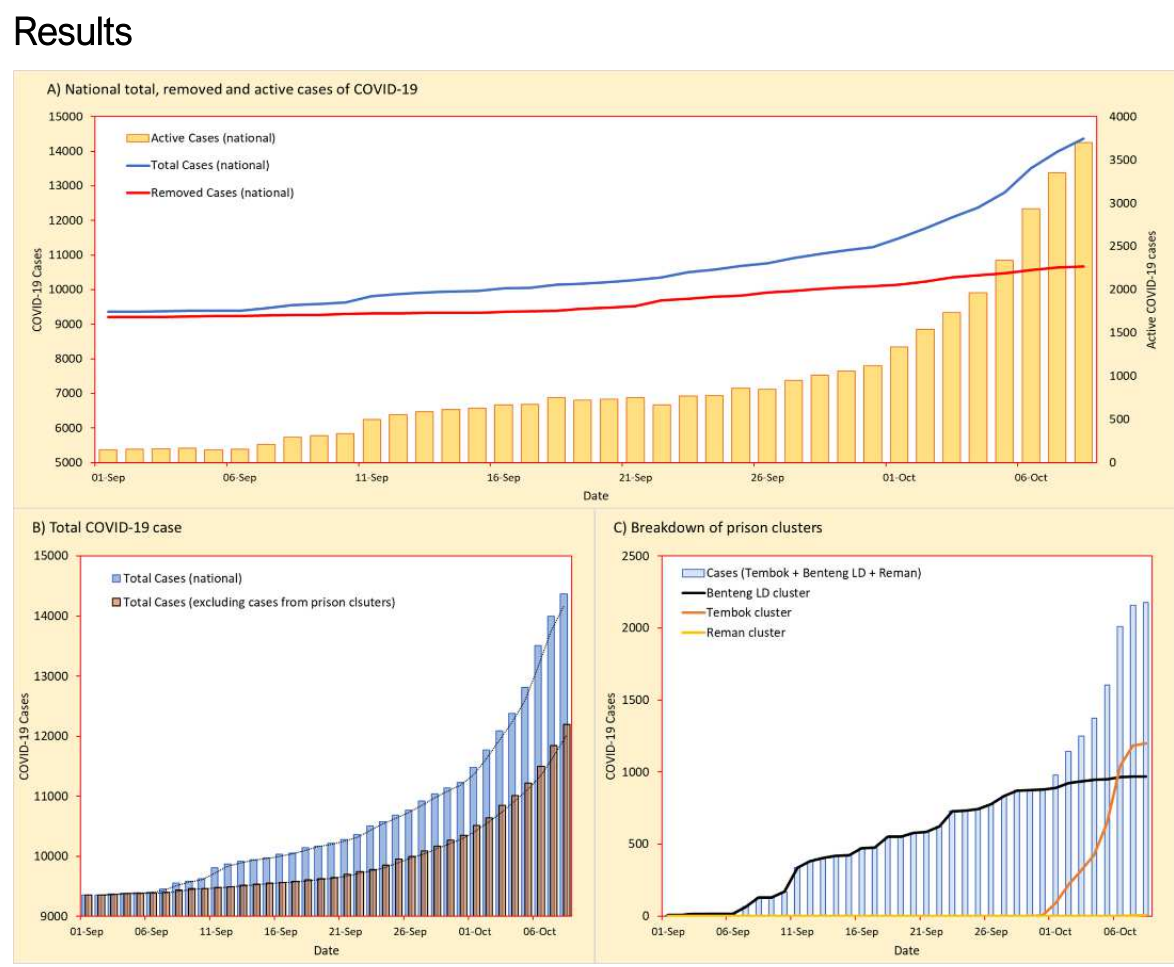

Figure 2: Actual cases of COVID-19 for the latest outbreak in Malaysia

A) Total, removed, and active cases of COVID-19 in Malaysia inclusive of incarcerated clusters, B) Total COVID-19 case with inclusion and exclusion of incarcerated cluster, and C) COVID-19 cases according to Benteng LD, Tembok, and Reman clusters up to 8 October 2020.

Figure 2 presented actual cases of COVID-19 of the latest outbreak in Malaysia starting from 1 September 2020 till 8 October 2020. Total, removed, and active cases of COVID-19, including incarcerated clusters such as the Benteng LD cluster, Tembok cluster and Reman cluster were presented in Figure 2A. Based on the break-ups of cases, we rebuilt the trendline of total cases of COVID-19 without cases from incarcerated clusters (Figure 2B). The inclusion of cases from incarcerated clusters significantly elevated the trendline of total cases for COVID-19 in the country, especially after 11 September 2020. Figure 2C presented cases according to the Benteng LD cluster $(\mathrm{N}=971)$, Tembok cluster ( $\mathrm{N}=1199)$, and Reman cluster $(\mathrm{N}=7)$ till 8 October 2020. The initial national trendline of COVID-19 was contributed only by the Benteng LD cluster, while the Tembok cluster and Reman cluster contributed to the latter part of the trendline.

Figure 3 presented the results of modelling with the inclusion of incarcerated clusters and projection without incarcerated clusters. The dynamics of transmission with the inclusion of incarcerated clusters was characterized by $\widehat{R}=2.0$ (Figure $3 \mathrm{~A}$ ), with a ratio of non-isolated active cases to isolated active cases increased slightly from 1.0 to 1.5 over 25 days (Figure 3B). Durations of transmission and hospitalization were estimated to be 10.5 days and 20 days, respectively. The duration of transmission in this outbreak was almost two times of what was reported in our previous study. ${ }^{8}$

Based on the duration of transmission and hospitalization, modelling was done without cases from incarcerated clusters. Notably, the $\widehat{\mathrm{R}}$ was increased to 2.3 (Figure 3D), with a ratio of nonisolated active cases to isolated active cases increased from 0.28 to 1.60 over 25 days. Th ratio had 
reached 1.76 as of 8 October 2020 (Figure 3E). The dynamics of transmission progressively increased without being noticed with the inclusion of incarcerated clusters in the trendline. As of 8 October 2020, total active cases were estimated to be 2.8 times of active cases isolated and reported by the health authority. Besides masking the dynamics of transmission with lower transmissibility, the inclusion of incarcerated cases also had introduced more variations and uncertainties into the trendline of daily cases (Figure $3 \mathrm{C}$ ), which made modelling inaccurate without excluding them from the reported data (Figure 3F).

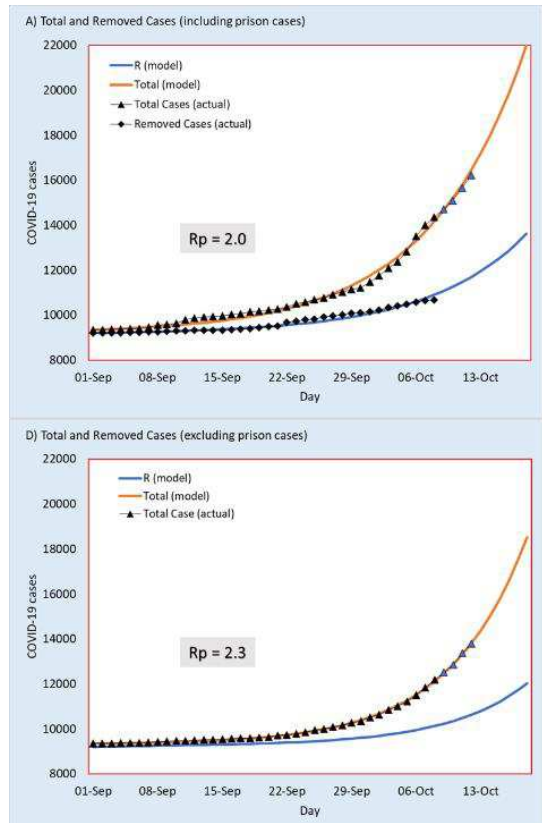

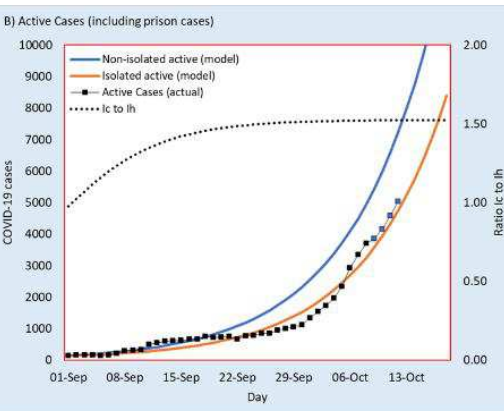

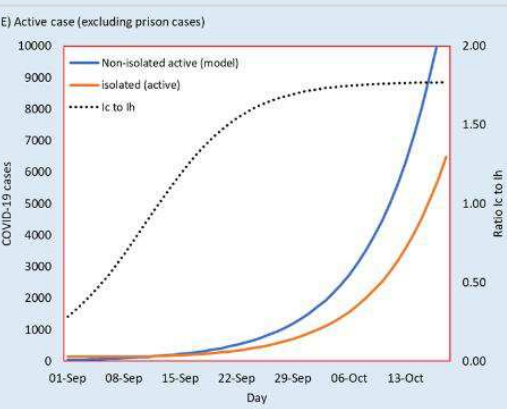

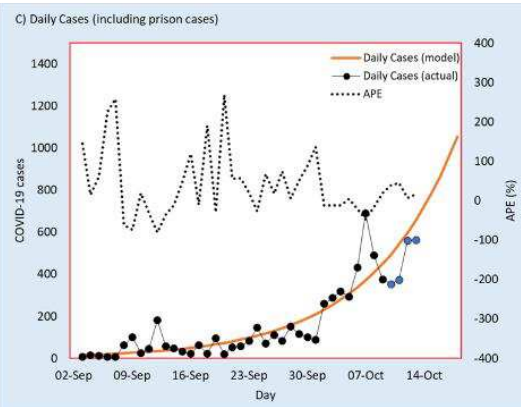

F) Daily Cases (excluding prison cases)

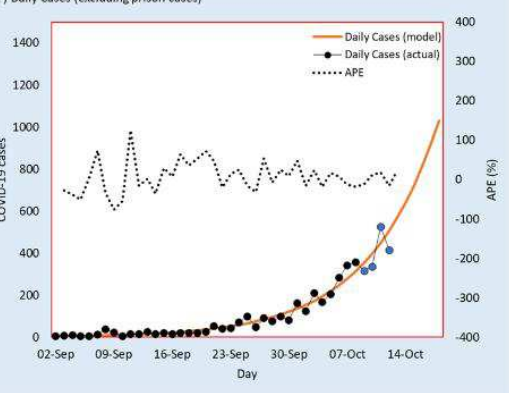

Figure 3: Model projections

With the inclusion of incarcerated clusters, A) The $\widehat{R}$ was estimated to be 2.0, B) Non-isolated active cases were similar to isolated active cases, and C) More variations were detected in actual daily cases. With the exclusion of incarcerated clusters, D) The $\widehat{R}$ was increased to 2.3 , E) Nonisolated active cases appeared to be much less in the beginning, and grew progressively to nearly two times of isolated active cases at the end of September, and F) Less variation was detected in the actual daily cases.

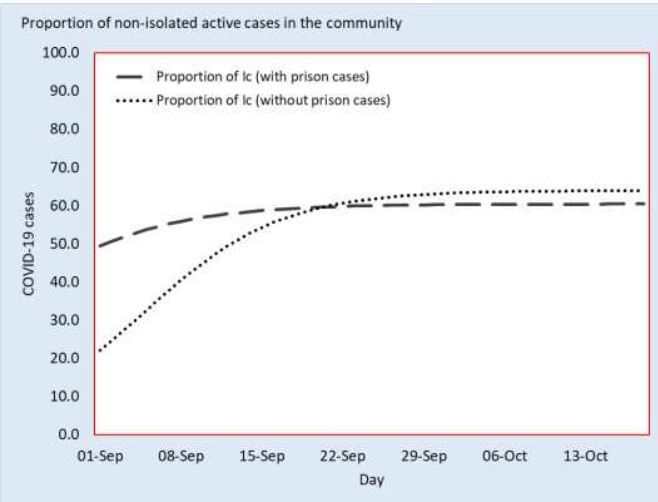

Figure 4: The proportion of non-isolated active cases in the community with and without incarcerated clusters. The buildup of non-isolated active cases in the community was increasing progressively but had been shadowed with the inclusion of incarcerated clusters. 
Figure 4 presented the growing proportion of non-isolated active cases over time with and without incarcerated clusters. The proportion of non-isolated active cases increased slightly from 49.4\% to $60.3 \%$ from 1 September 2020 to 8 October 2020 (10.9\% increment over 38 days) with the inclusion of incarcerated clusters. More progressive growth of non-isolated active cases was found, $22.1 \%$ to $63.7 \%$ from 1 September 2020 to 8 October 2020 (41.6\% increment over 38 days) without incarcerated clusters. The progressive growth of non-isolated active cases, which was driving the widespread of COVID-19 in the community could not be clearly captured with the inclusion of incarcerated clusters in the trendline.

Table 1 summarized characteristics of transmission for COVID-19 with and without incarcerated clusters. As of 8 October 2020, COVID-19 cases originated from incarcerated clusters accounted for $56.7 \%$ of the total cases reported. Only 2179 (43.3\%) cases were considered cases in the community. The dynamics of transmission for COVID-19 could be underestimated with the inclusion of incarcerated clusters, especially in the growth of non-isolated active cases.

Table 1: The dynamics of transmission for COVID-19 with and without incarcerated clusters.

\begin{tabular}{|l|c|c|c|}
\hline \multirow{2}{*}{ Characteristics (As of 8 October 2020) } & \multirow{2}{*}{ Definition } & \multicolumn{2}{|c|}{ Incarcerated clusters } \\
\cline { 3 - 4 } & & With & Without \\
\hline Total confirmed cases & Total Ih & 5028 & $2849(56.7 \%)$ \\
Isolated active cases & Ih & 3703 & 1989 \\
Non-isolated active cases & IC & 5628 & 3494 \\
$\widehat{\mathrm{R}}$ & $\beta / \sigma$ & 2.0 & 2.3 \\
Proportion of non-isolated active cases & Ic / (Ic + Ih) & & \\
- On 1 September 2020 & & 49.4 & 22.1 \\
- On 8 October 2020 & IC : Ih & 1.5 & 63.7 \\
Ratio of Ic to Ih & (Ic + Ih )/lh & 2.5 & 1.8 \\
Total active cases over reported active cases & & & 2.8 \\
\hline
\end{tabular}

Table 2 presented the MAPE \pm standard deviation (SD) in percentage (\%) for model fitting with actual cases from 1 September 2020 to 8 October 2020. Incarcerated clusters increased the MAPE significantly and may lead to more uncertainties in modelling. The MAPE was reduced by $16.84 \%$ and $0.97 \%$ for daily cases and total cases by excluding incarcerated clusters in modelling. Table 3 presented the MAPE \pm SD for model projection with actual cases from 9 October 2020 to 15 October 2020. Similarly, projections were more accurate without incarcerated clusters.

Table 2: MAPE for model fitting from 1 September 2020 to 8 October 2020.

\begin{tabular}{lcc}
\hline \multirow{2}{*}{ State variables } & \multicolumn{2}{c}{ MAPE \pm SD (\%) } \\
\cline { 2 - 3 } & With incarcerated clusters & Without incarcerated clusters \\
\hline Daily new cases & $56.48 \pm 79.10$ & $39.64 \pm 54.41$ \\
Total cases & $1.25 \pm 0.81$ & $0.28 \pm 0.21$ \\
Active cases & $26.13 \pm 22.38$ & - \\
Removed cases & $0.63 \pm 0.67$ & - \\
\hline
\end{tabular}


Table 3: MAPE for model projection from 9 October to 15 October 2020.

\begin{tabular}{lcc}
\hline \multirow{2}{*}{ State variables } & \multicolumn{2}{c}{ MAPE \pm SD $(\%)$} \\
\cline { 2 - 3 } & With incarcerated clusters & Without incarcerated clusters \\
\hline Daily new cases & $19.67 \pm 10.12$ & $16.51 \pm 7.84$ \\
Total cases & $1.62 \pm 1.08$ & $0.74 \pm 0.79$ \\
Active cases & $4.90 \pm 2.42$ & - \\
Removed cases & $3.24 \pm 0.73$ & - \\
\hline
\end{tabular}

\section{Discussion}

The second outbreak of COVID-19 in Malaysia happened in early March 2020. It was driven by a large religious gathering event involving more than 14000 attendees from different countries, including countries that were severely hit by COVID-19 like South Korea. The exponential surge of COVID-19 cases was characterized by a high $\widehat{\mathrm{R}}_{0}(2.26-3.50)$, depending on the duration of infectiousness ( 5.5 days - 8.5 days). ${ }^{8}$ The surge urged the Federal Government of Malaysia to impose a nationwide MCO to contain the widespread of COVID-19 in the country. During the MCO, stringent public health measures were enforced, and the epidemic curve of COVID-19 was flattened successfully in April 2020, a month after the surge. After that, the daily new cases of COVID-19 had subsided to single-digit cases at the end of July 2020. That outbreak took the nation nearly four months to end with billions of financial payouts to sustain the economic resilience during the lockdown period.

Unlike the second wave of COVID-19, the latest outbreak was complicated by the occurrence of multiple incarcerated clusters in Tawau, Lahad Datu, Alor Setar and Penang. In Malaysia, incarcerated populations posted minimal threats to the community as they were separated from the community during their disease duration. A large amount of incarcerated cases might have introduced a false impression to the authority that the outbreak of COVID-19 in the community was still containable. This might explain a delay in implementing travel restriction and compulsory quarantine on returnees from Sabah before the outbreak worsened.

The Sabah state by-election and more frequent inter-state travels following the end of the second wave of COVID-19 were believed to be the main reasons for the widespread of COVID-19 in the community again. However, the trendline of COVID-19 had been uplifted unevenly due to the inclusion of incarcerated clusters. The uplift reduced the slope of the trendline and prevented the progressively growing dynamics of transmission in the community from being visualized through modelling.

The latest outbreak without incarcerated clusters was characterized by a $\widehat{\mathrm{R}}_{0}$ of 2.3 with a longer duration of transmission (10.5 days). The non-isolated active cases of COVID-19 may consist of those in incubation period, asymptomatic and mild symptomatic but were able to transmit the coronavirus. This class was estimated to be 1.8 times of isolated active cases as of 8 October 2020, 6 times bigger than the ratio identified on 1 September 2020. A longer duration of transmission and a significantly increased of non-isolated active cases in the community helped explained the worsening COVID-19 outbreak in the community even the $\widehat{\mathrm{R}}_{0}$ was smaller than before. 
In the absence of effective treatment for COVID-19, public health measures remained useful and effective in preventing and controlling the spread of COVID-19 in the community. The authority should consider enhancing the contract tracking coverage and screening capacity to isolate more infectious or active cases in the community. This helped fastened the transition of individuals from Ic to $\mathrm{Ih}$, and then a decline in contact rate and duration of transmission.

Confined quarters like jails and prisons has been shown to be a viral hotspot in several studies with a higher rate of transmission and severe outcomes, including death. ${ }^{6,9}$ Even with a higher rate of transmission, the presence of incarcerated clusters might not necessarily alter the dynamics of transmission in the community with a higher reproduction number. In our situation, a reverse outcome was found. The inclusion of incarcerated cases in the reported data uplifted the epidemic curve of COVID-19 and introduced more variations into it, contributed to a falsely lower transmission rate, a slower growth in non-isolated active cases.

Like many modelling studies utilizing reported data, our study might be affected by uneven screening and reporting rate. Screening effort in Sabah was limited by available resources and had caused a delay in testing and reporting cases to the authority. Clearing up the testing backlog might contribute to a sudden surge in daily cases. ${ }^{2}$ Another limitation was that incarcerated clusters could not be removed entirely from the modelling system as break-ups of cases according to clusters were not available for recovery and death cases. Besides, we assumed incarcerated clusters included mainly prisoners and detainees, while in fact, those clusters might include a substantial amount of correctional staff, nurses, chaplains, wardens and other workers, and their family members.

\section{Conclusion}

The dynamics of transmission of COVID-19 in the community was underrated and misjudged with the inclusion of incarcerated clusters. The progressive growth of non-isolated active cases was believed to impel the latest outbreak of COVID-19 and was seriously underestimated with the occurrence of multiple incarcerated clusters at different time. More resources should be allocated to enhance the radius and speed of contact tracking and screening in order to reduce non-isolated active cases in the community, as this class of individuals was believed to be the determinant for transmission in the community.

\section{Acknowledgements}

The author would like to thank the Crisis Preparedness and Response Centre for providing relevant data required in this study. The author would like to thank the Director-General of Health Malaysia for his permission to publish this manuscript.

\section{Author contributions}

All authors conceived and planned the study and contributed to the design of the mathematical model and interpretation of findings. LKB conducted the data analysis. All authors contributed to this manuscript and approved the final version for publication.

\section{Declaration of interest}

All authors declare no competing interest.

\section{Funding}


No source of funding.

\section{Ethics requirement}

The study has been registered with the National Medical Research Register. No ethics approval is required.

\section{References}

1. Boo S-L. Politicians Who Didn't Quarantine 14 Days After Sabah Travel (And Those Who Are). CodeB/ue. https://codeblue.galencentre.org/2020/10/02/politicians-who-didnt-quarantine-14days-after-sabah-travel-and-those-who-are/. Published October 2, 2020.

2. Situation Report of COVID-19 In Malaysia (Https://Kpkesihatan.Com/). MOH Malaysia

3. D Kanyakumari. "No total lockdown as yet", says PM Muhyiddine as COVID-19 continue to spike. Channe/ News Asia. https://www.channelnewsasia.com/news/asia/malaysia-pmmuhyiddin-no-total-lockdown-covid-19-cases-spike-13211118. Published October 6, 2020. Accessed October 8, 2020.

4. Franco-Paredes C, Jankousky K, Schultz J, et al. COVID-19 in jails and prisons: A neglected infection in a marginalized population. PLoS Neg/ Trop Dis. 2020;14(6):e0008409. doi:10.1371/journal.pntd.0008409

5. Hawks L, Woolhandler S, McCormick D. COVID-19 in Prisons and Jails in the United States. JAMA Intern Med. 2020;180(8):1041-1042. doi:10.1001/jamainternmed.2020.1856

6. Jiménez MC, Cowger TL, Simon LE, Behn M, Cassarino N, Bassett MT. Epidemiology of COVID-19 Among Incarcerated Individuals and Staff in Massachusetts Jails and Prisons. JAMA Netw Open. 2020;3(8):e2018851-e2018851. doi:10.1001/jamanetworkopen.2020.18851

7. Akiyama MJ, Spaulding AC, Rich JD. Flattening the Curve for Incarcerated Populations Covid-19 in Jails and Prisons. N Eng/ J Med. 2020;382(22):2075-2077. doi:10.1056/NEJMp2005687

8. Law KB, Peariasamy K. Predicting the early depleting transmission dynamics of COVID-19: A time-varying SIR model.

9. Lofgren E, Lum K, Horowitz A, Madubuonwu B, Fefferman N. The Epidemiological Implications of Incarceration Dynamics in Jails for Community, Corrections Officer, and Incarcerated Population Risks from COVID-19. medRxiv. Published online January 1 , 2020:2020.04.08.20058842. doi:10.1101/2020.04.08.20058842 
Figures

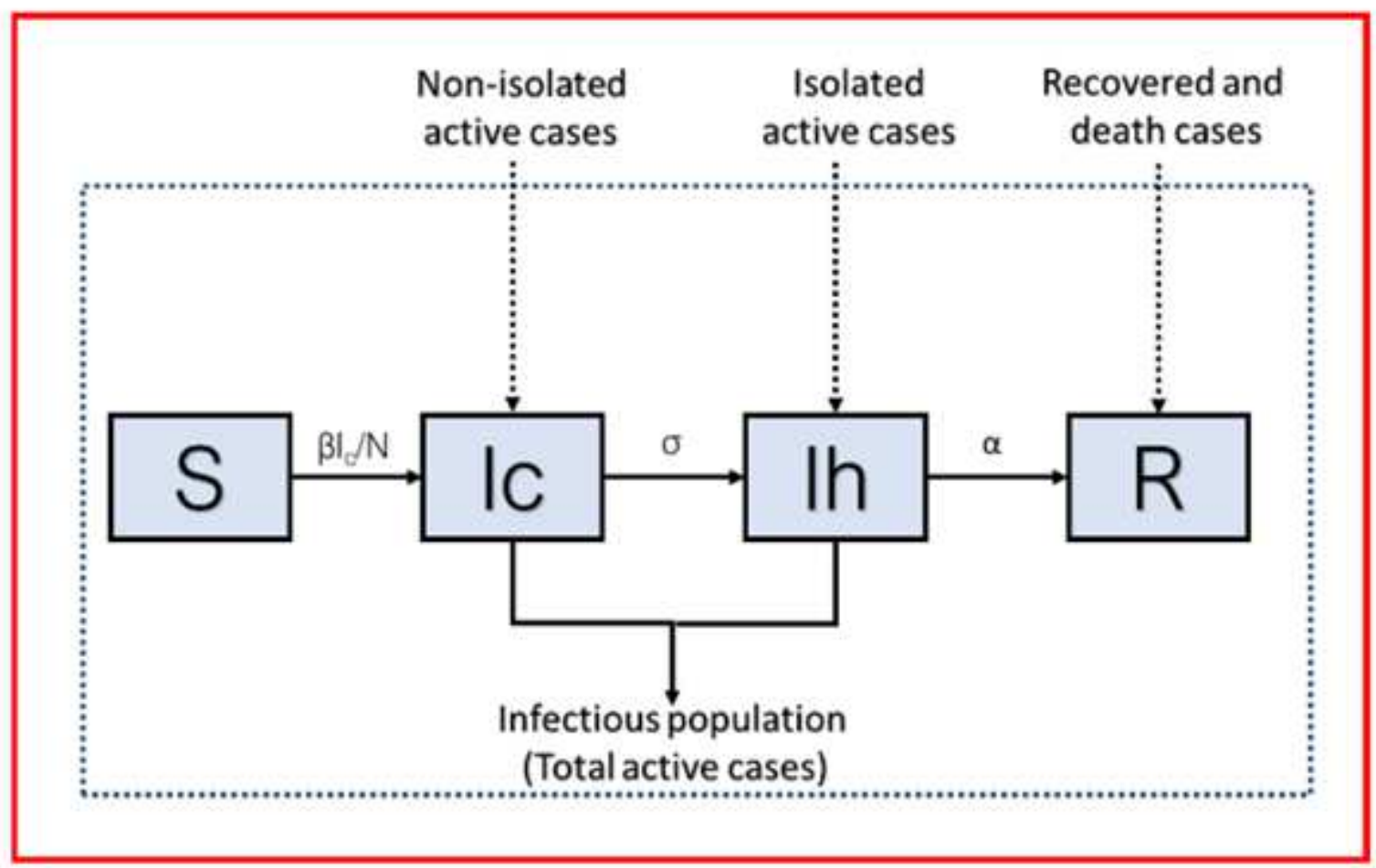

Figure 1

Model structure The infectious class was divided into non-isolated infectious and isolated infectious. Non-isolated infectious was assumed to be the determinant for the spread of coronavirus in the community. 


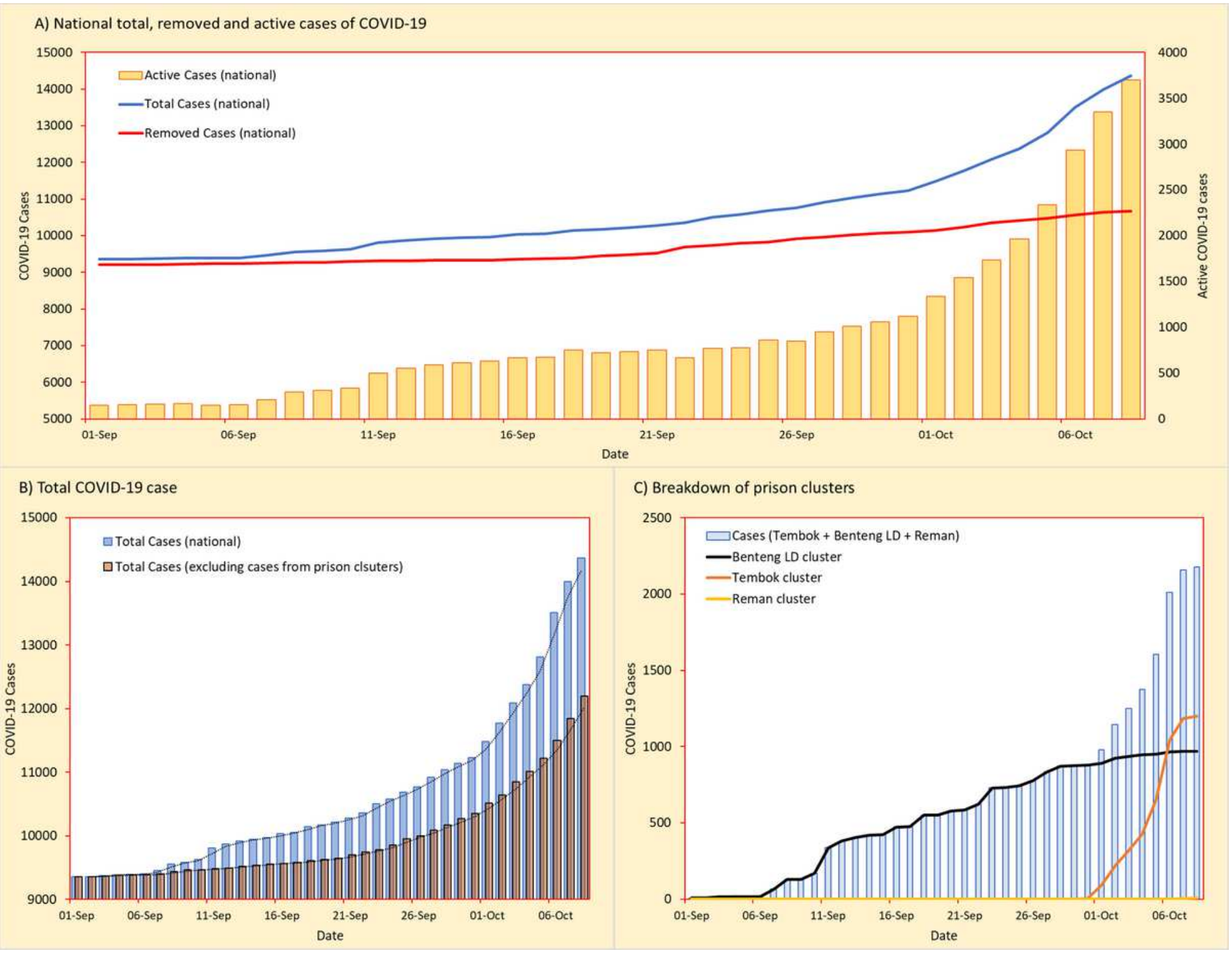

Figure 2

Actual cases of COVID-19 for the latest outbreak in Malaysia A) Total, removed, and active cases of COVID-19 in Malaysia inclusive of incarcerated clusters, B) Total COVID-19 case with inclusion and exclusion of incarcerated cluster, and C) COVID-19 cases according to Benteng LD, Tembok, and Reman clusters up to 8 October 2020. 

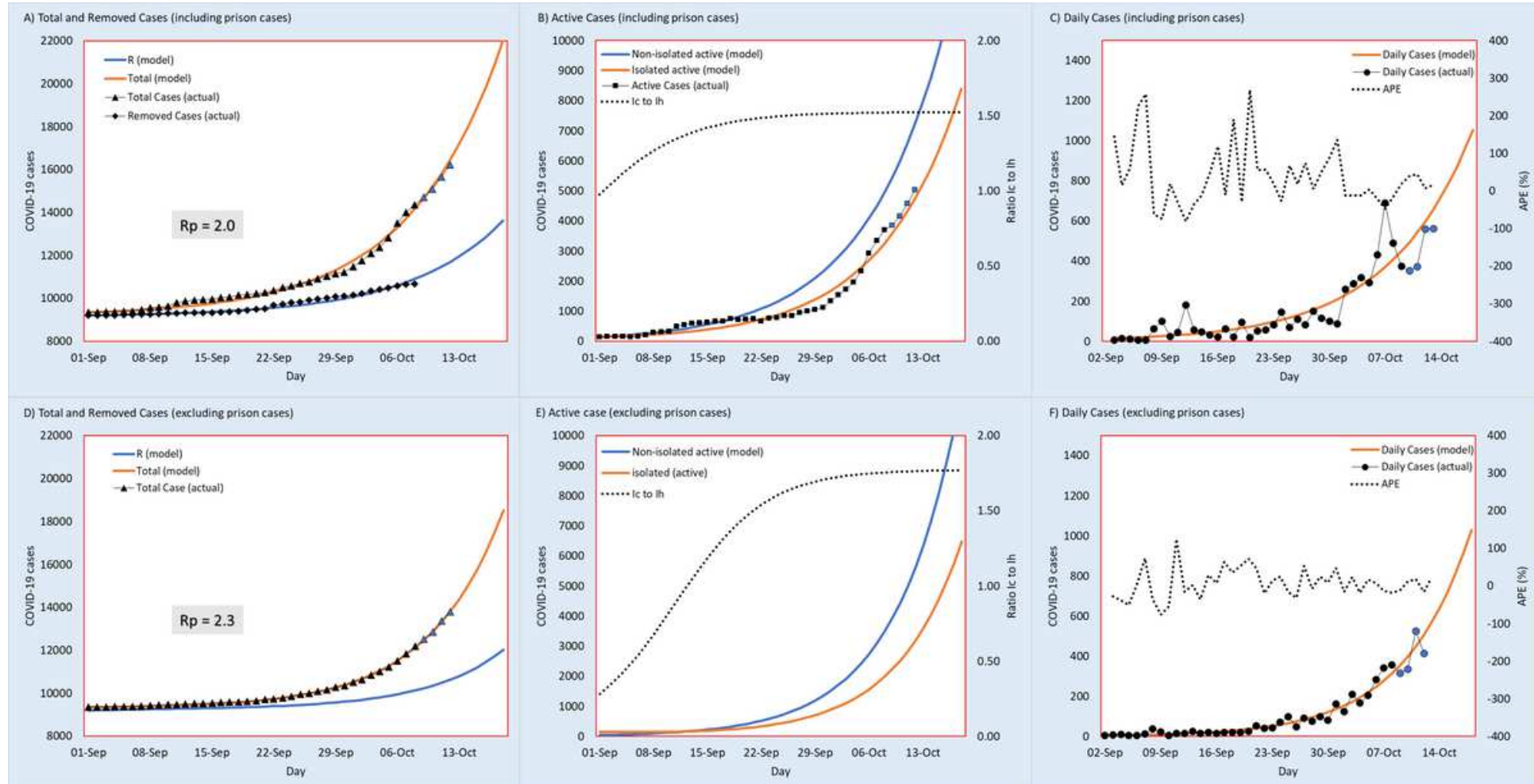

Figure 3

Model projections With the inclusion of incarcerated clusters, A) The R $\otimes$ was estimated to be 2.0, B) Nonisolated active cases were similar to isolated active cases, and C) More variations were detected in actual daily cases. With the exclusion of incarcerated clusters, D) The R $\otimes$ was increased to 2.3, E) Non-isolated active cases appeared to be much less in the beginning, and grew progressively to nearly two times of isolated active cases at the end of September, and F) Less variation was detected in the actual daily cases. 
Proportion of non-isolated active cases in the community

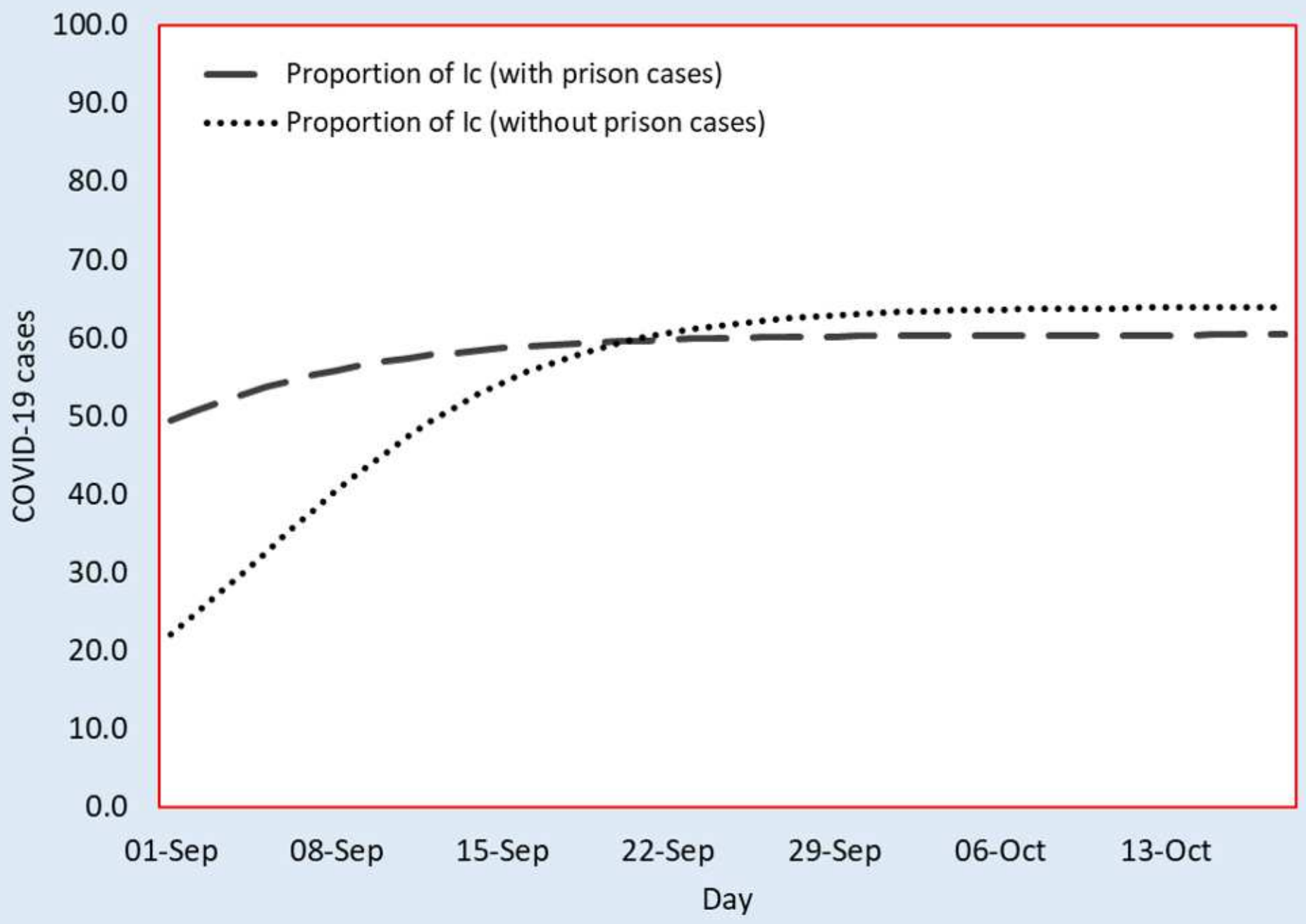

Figure 4

The proportion of non-isolated active cases in the community with and without incarcerated clusters. The build-up of non-isolated active cases in the community was increasing progressively but had been shadowed with the inclusion of incarcerated clusters. 